\title{
Genetic expression profile-based screening of genes and pathways associated with papillary thyroid carcinoma
}

\author{
SHUBIN $\mathrm{LI}^{1}$, YIHANG YIN ${ }^{2}$ and HONG YU ${ }^{3}$ \\ ${ }^{1}$ Department of Internal Medicine, Southern Branch of Guang'anmen Hospital, China Academy of Chinese Medical Sciences, \\ Beijing 102600; ${ }^{2}$ School of Computer Science and Engineering, Beihang University, Beijing 100191; ${ }^{3}$ Cell Biology Laboratory, \\ Jilin Province Institute of Cancer Prevention and Treatment, Jilin Cancer Hospital, Changchun, Jilin 130012, P.R. China
}

Received October 9, 2017; Accepted July 27, 2018

DOI: $10.3892 / \mathrm{ol} .2018 .9342$

\begin{abstract}
Papillary thyroid carcinoma (PTC) is the most common subtype of thyroid cancer; however, the specific genes and signaling pathways involved in this cancer remain largely unclear. The present study analyzed three profile datasets, GSE6004, GSE29265 and GSE60542, which were comprised of 47 PTC and 41 normal thyroid tissue samples, to identify key genes and pathways associated with PTC. Initially, differentially-expressed genes (DEGs) between PTC and normal thyroid tissue were screened using R 3.4.0 (2017-04-21, R Foundation, Vienna, Austria, https://www.R-project.org/). These DEGs were then clustered by gene ontology functional terms and representative signaling pathways. Additionally, specific key gene nodes were filtered out from a constructed protein-protein interaction (PPI) network. The results identified a total of 423 shared DEGs associated with PTC, including 211 upregulated and 212 downregulated genes. These 423 genes were primarily enriched in glycosaminoglycan binding, sulfur compound binding, heparin binding, enzyme activator activity, peptidase activator activity and hsa04512: Extracellular matrix (ECM)-receptor interaction. A total of 21 central node genes were identified as key genes in the PTC disease process including complement factor D (CFD), Collagen Type I $\alpha 1$ Chain (COL1A1), Extracellular Matrix Protein 1 (ECM1) and Fibronectin 1 (FN1). These genes are involved in protease binding, G-protein coupled receptor binding, extracellular matrix structural constituent and peptidase regulator activity. To conclude, using bioinformatics analysis, the present study identified candidate DEGs and critical pathways in PTC that may improve the current
\end{abstract}

Correspondence to: Dr Hong Yu, Cell Biology Laboratory, Jilin Province Institute of Cancer Prevention and Treatment, Jilin Cancer Hospital, 1018 Huguang Road, Changchun, Jilin 130012, P.R. China E-mail: yhzhlyy@126.com

Key words: papillary thyroid carcinoma, differentially expressed genes, signaling pathways, extracellular matrix, collagen, bioinformatics analysis understanding regarding the underlying mechanisms of PTC. These genes and pathways may be used as potential therapeutic targets of PTC in the future.

\section{Introduction}

Thyroid cancer is the most common type of endocrine malignancy in the United States in 2015 (1). In China, thyroid carcinoma resulted in 6,800 mortalities and 90,000 new cases in 2015 (2). Although clinical practice guidelines for this disease have been updated in recent years, the morbidity of this disease has not changed (3). For females in particular, the disease burden for thyroid cancer with uncertain etiology is increased due to the increased disease incidence in females, which is 3 times higher than that of males ( 21 vs. 7 per 100,000 population), albeit with a comparable mortality ( 0.5 per 100,000 population) (4). Although numerous studies have used next-generation sequencing and other novel technologies to investigate the potential key genes and pathways in thyroid cancer, its key molecular mechanisms remain unclear (5-9). Elucidating the underlying mechanisms in thyroid cancer is crucial for the diagnosis and prevention of this deadly disease.

Gene chip and gene expression profiles can be used to analyze the total genetic information within a sample, thus making them appropriate for differentially expressed gene screening. In parallel with extended gene detection applications, numerous microarray data have been generated and deposited into public databases (5-7). Re-analysis of these data can offer novel insights regarding specific gene expression in disease (10). Unlike conventional experimental research, these re-analysis articles focus on bioinformatics analysis to screen differentially-expressed genes (DEGs), construct gene functional networks, and identify novel targets for disease diagnosis and treatment (11). At present a plethora of bioinformatics articles have been published, especially pertaining to cancer research $(12,13)$. With regard to thyroid cancer, multiple studies have described hundreds of DEGs and signaling pathways $(8,10,11,14-21)$. However, these results are always circumscribed or inconsistent with each other due to heterogeneity between study samples, or the fact that they were generated from single cohort studies $(11,16,21)$. Consequently, a comprehensive analysis of the integrated gene expression 
data with bioinformatic methods is a superior method to overcome these previous shortcomings.

Thyroid cancer may be categorized into multiple subtypes. Among them, the most common subclass is papillary thyroid carcinoma (PTC), comprising $80 \%$ of total thyroid carcinomas (20). In the present study, three original datasets GSE6004 (22), GSE29265 and GSE60542 (23) from the NCBI-Gene Expression Omnibus database (NCBI-GEO) (https://www.ncbi.nlm.nih.gov/geo) (24) were processed to identify the DEGs between PTC tissue and healthy tissue controls. The present study further filtered DEGs and completed gene ontology (GO) and pathway enrichment via limma (25) package and clusterProfiler in $\mathrm{R}$ 3.4.0 (2017-04-21, R Foundation, Vienna, Austria) (26) and Panther (http://www.pantherdb.org) (27). A protein-protein interaction (PPI) network (http://string-db.org) (28) of DEGs was constructed along with modular analysis to identify key genes in PTC. The results of the present study may provide more practically precise and credible biomarkers for use in the diagnosis, prevention and individualized therapy of PTC.

\section{Materials and methods}

Data preparation and DEG identification. From NCBI-GEO (https://www.ncbi.nlm.nih.gov/gds/), three gene expression datasets of GSE6004 (22), GSE29265 and GSE60542 (23) were selected if they met four criteria: i) The deposited raw data were in CEL format; ii) the datasets contained PTC and paired healthy thyroid tissue samples. iii) GSE datasets involved in published papers were excluded; iv) Chernobyl-related specimens were removed from the remaining eligible samples based on the conclusion that these PTCs are different from those sporadic PTCs, as the present study wanted to analyze the latter (8). These three datasets were all analyzed on the GPL570 platform (Affymetrix human genome U133 plus 2.0 array, Affymetrix; Thermo Fisher Scientific, Inc., Waltham, MA, USA) (http://www.affymetrix.com/support/technical/byproduct.affx?product=hg-u133-plus). Detailed sample information on these microarrays are listed in Table I. The raw data of these datasets were processed by R 3.4.0 (2017-04-21, R Foundation, Vienna, Austria) using the 'affy' package (29) and 'limma' package (25) for variance stabilization, background correction, normalization and log transformation. DEGs were defined based on the criteria $\mathrm{p} P<0.01$ and $\mid \log \mathrm{FCl}>1.5$. For validation, PTC data, including 58 normal and 356 thyroid cancers, were obtained from The Cancer Genome Atlas (TCGA) (https://cancergenome.nih.gov/) and analyzed (20).

Hierarchical analysis of GO and pathways of DEGs. The GO and Kyoto Encyclopedia of Genes and Genomes (KEGG) pathways of DEGs were hierarchically investigated using 'clusterProfiler' in R 3.4.0 (2017-04-21, R Foundation, Vienna, Austria) (26) and Panther (http://www.pantherdb.org) (27), a website that can visualize integrated gene information.

Constructing PPI network and modular analysis. The PPI network of PTC-associated DEGs-encoded proteins was constructed using STRING (http://string-db.org) (30). Decisive candidate proteins in prominent modules (confidence score of $>0.9$ ) with pivotal physiologic regulation of PTC were
Table I. Data descriptions of the three datasets from GEO.

\begin{tabular}{lccc}
\hline $\begin{array}{l}\text { GSE } \\
\text { number }\end{array}$ & $\begin{array}{c}\text { Tumor } \\
\text { tissue }\end{array}$ & $\begin{array}{c}\text { Normal } \\
\text { control }\end{array}$ & $\begin{array}{c}\text { Submission } \\
\text { date }\end{array}$ \\
\hline GSE6004 & 7 & 4 & Oct 10, 2006 \\
GSE29265 & 7 & 7 & May 31, 2011 \\
GSE60542 & 33 & 30 & Aug 20, 2014 \\
Total & 47 & 41 & \\
\hline
\end{tabular}

GEO, gene expression omnibus.

retrieved by Cytoscape software (version 3.5.1, The Cytoscape Consortium, San Diego, CA, USA) (http://www.cytoscape. $\operatorname{org} /)(31)$.

Reverse transcription-quantitative polymerase chain reaction (RT-qPCR) assay. In the present study, 8 DEGs (5 upregulated and 3 downregulated) were selected for validation in peripheral blood samples of PTC patients using RT-qPCR. The present study was approved by the Ethics Committee of Guang'anmen Hospital (Beijing, China). Patients provided written informed consent prior to blood collection. From March 1st, 2018 to March 31st, 2018, peripheral blood samples of 21 PTC patients ( $2 \mathrm{ml} / \mathrm{sample}$ ) were collected from the department of internal medicine at the Southern branch of Guang'anmen Hospital. PTC patients were selected based on two criteria: First hospitalized and no other autoimmune disease or other malignancies at the time of the investigation (age range, 26 to 65 years; median age, 46.5 years; 4 males, 17 females). Then 21 blood samples from healthy people ( $2 \mathrm{ml} / \mathrm{sample})$ served as the control (age range, 20 to 63 years; median age, 47.5 years; 4 males, 17 females). The PCR primers were as follows: Forward primer AGTR1, 5'-TTGTTGAAAGGTTTGAGTGGG-3' and reverse primer AGTR1, 5'-TTGCAGATATTGTGGACACGG-3'; CFD forward primer, 5'-CGATGGTGTCGGGCTGGCTGT-3' and reverse primer, 5'-GCCCTACATGGCGTCGGTGCA-3'; COL1A1 forward primer, 5'-CGATGGATTCCAGTTCGA GTATG-3' and reverse primer, 5'-TGTTCTTGCAGTGGT AGGTGATG-3'; COL8A1 forward primer, 5'-CTTTCTGTC CAATTTCTCCTT-3' and reverse primer, 5'-ATACCATTA GCCAGTTTACGA-3'; COL10A1 forward primer, 5'-GAT GATGGCACTCCCTGAAGC-3' and reverse primer, 5'-ATA AGA ATGGCACCCCTGTAA-3'; LPAR5 forward primer, 5'-ACGGCGGACCTTTCGGATTGC-3' and reverse primer, 5'-GCGGGGTGCTGATGGTGATGG-3'; MMRN1 forward primer, 5'-ATGGGCAGGAAGTCTGGTTACGA-3' and reverse primer, 5'-ACAGAGCAGATGTGCAAGCAA AGAT-3'; NMU forward primer, 5'-CATTCCCATAATCAT AAAGCAA-3', reverse primer, 5'-AAGGATTACAGCCTG AACAAC-3'; $\beta$-actin forward primer, 5'-CTACCTCATGAA GATCCTCACCGA-3', reverse primer, 5'-TTCTCCTTAATG TCACGCACGATT-3'.

Total RNA was isolated from blood samples using TRIzol reagent (Invitrogen; Thermo Fisher Scientific, Inc.). Reverse transcription and real-time quantification were performed using PrimeScript ${ }^{\mathrm{TM}}$ RT reagent kit with gDNA Eraser (Perfect Real Time) (Takara Biotechnology Co., Ltd., Dalian, 


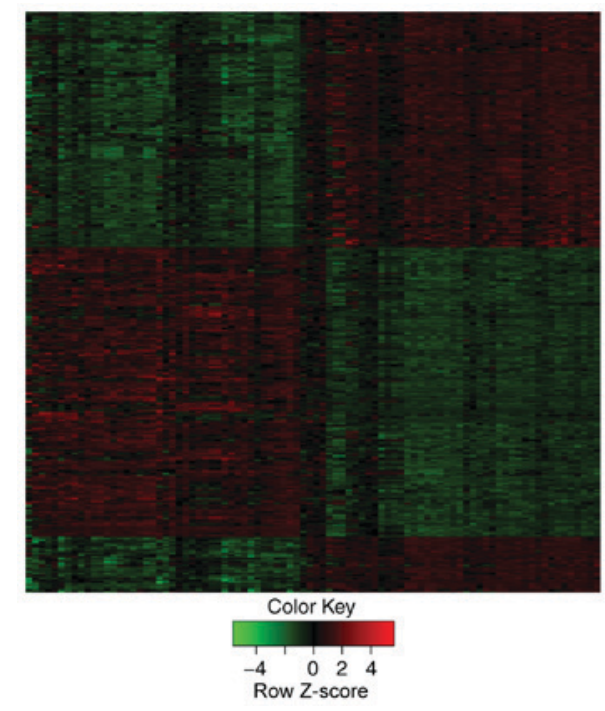

Figure 1. Expression heat map of 423 differentially expressed genes. The $\mathrm{x}$ axis indicates the samples and the $\mathrm{y}$ axis indicates the DEGs. DEGs, differentially-expressed genes.

China) and TB Green ${ }^{\mathrm{TM}}$ Premix Ex Taq ${ }^{\mathrm{TM}}$ (Tli RNaseH Plus) (Takara Biotechnology Co., Ltd., Dalian, China) according to manufacturer's protocol (32). Each measurement was made in triplicate and normalized with $\beta$-actin control. The thermal cycling conditions included an initial denaturing step at $95^{\circ} \mathrm{C}$ for $30 \mathrm{sec}, 40$ cycles at $95^{\circ} \mathrm{C}$ for $5 \mathrm{sec}, 60^{\circ} \mathrm{C}$ for $30 \mathrm{sec}$. The relative gene expression data were analyzed using $2^{-\Delta \Delta \mathrm{Cq}}$ algorithm (33) according to the literature (34) and the RDML (Real-Time PCR Data Markup Language) data standard (http://www.rdml.org) (35).

Statistical analysis. Statistical analyses were performed with GraphPad Prism 5 (GraphPad Software, Inc., La Jolla, CA, USA). Data from the qPCR experiments are presented as mean \pm standard deviation. The significance of differential expression between PTCs and controls was assessed by an unpaired Student's t-test. $\mathrm{P}<0.05$ was considered to indicate a statistically significant difference.

\section{Results}

Screening of DEGs from PTC and paired thyroid tissues based on NCBI-GEO datasets. Employing the cut-off criteria $\mathrm{P}<0.01$ and $\mid \log \mathrm{FCl}>1.5,423$ DEGs were identified from PTC and paired healthy thyroid tissues based on three datasets GSE6004, GSE29265 and GSE60542 (data not shown) using R 3.4.0 (2017-04-21; R Foundation, Vienna, Austria) program. Among these genes, up- and downregulated genes accounted for 211 and 212 genes, respectively. These DEGs could be clearly discriminated between PTC and normal controls with heat map visualization (Fig. 1).

Gene Ontology analysis of DEGs in PTC. Candidate DEGs gene ontology (GO) analysis was performed using the online database Panther (http://www.pantherdb.org) and R 3.4.0 (2017-04-21, R Foundation, Vienna, Austria) program (26). The DEGs were classified into molecular function (MF), biological process (BP) and cellular component (CC) groups
(Fig. 2). In the BP grouping, DEGs were mainly enriched in cellular component organization or biogenesis, localization, reproduction, and regulation. In the MF grouping, DEGs were mainly enriched in binding, receptor activity, structural molecule activity, signal transducer activity. In the CC grouping, DEGs were mainly enriched in synapse, cell junction, membrane, macromolecular complex, extracellular matrix.

Additionally, the present study used 'clusterProfiler' in R 3.4.0 (2017-04-21, R Foundation, Vienna, Austria) (26) to perform DEGs GO analysis. The results indicated that the majority of DEGs were clustered in glycosaminoglycan binding, sulfur compound binding, heparin binding, enzyme activator activity and peptidase activator activity (Fig. 3 and Table II). Upregulated DEGs were significantly enriched serine-type endopeptidase activity, serine-type peptidase activity, serine hydrolase activity, endopeptidase activity, protease binding. Unexpectedly, there were no downregulated DEGs involved in typical GO biological processes and pathways.

Screening of typical signaling pathways associated with $D E G s$. The typical signaling pathways associated with our DEGs were identified using 'clusterProfiler' in R 3.4.0 (2017-04-21, R Foundation, Vienna, Austria) (26). The DEGs had commonalities in hsa04512: ECM-receptor interaction pathway (Fig. 4A). The outcome of a reactome enquiry suggested that the majority of the DEGs were involved in extracellular matrix organization, degradation of the extracellular matrix, integrin cell surface interactions, collagen degradation, ECM proteoglycans (Fig. 4B and Table III). The reactome network is illustrated in Fig. 5.

PPI network, key nodes analyses and pathway identification. The PPI network was constructed based on 423 selected DEGs using STRING database. Furthermore, we filtered three prominent modules from it by Cytotype MCODE of Cytoscape depending on the importance of each gene (Fig. 6). These modules consisted of 21 genes: Complement factor D (CFD), collagen type $\mathrm{X} \alpha 1$ chain (COL10A1), collagen type XIII $\alpha 1$ chain (COL13A1), collagen type I $\alpha 1$ chain (COL1A1), collagen type XXIII $\alpha 1$ chain (COL23A1), collagen type VIII $\alpha 1$ chain (COL8A1), collagen type VIII $\alpha 2$ chain (COL8A2), collagen type IX $\alpha 3$ chain (COL9A3), Extracellular Matrix Protein 1 (ECM1), Fibronectin 1 (FN1), Multimerin 1 (MMRN1), Protein S (PROS1), Serpin Family A Member 1 (SERPINA1), TIMP Metallopeptidase Inhibitor 1 (TIMP1), Angiotensin II Receptor Type 1 (AGTR1), Arginine Vasopressin Receptor 1A (AVPR1A), Endothelin 3 (EDN3), G Protein Subunit $\alpha 14$ (GNA14), KISS1 Receptor (KISS1R), Lysophosphatidic Acid Receptor 5 (LPAR5) and Neuromedin U (NMU). Enrichment analysis (Table IV) demonstrated that these three modules were principally associated with protease and G-protein coupled receptor binding, extracellular matrix components and peptidase regulator activity.

TCGA datasets used to verify selection of 423 DEGs. To confirm the 423 DEGs screened in the current study, PTC datasets containing 356 PTC and 58 normal controls 


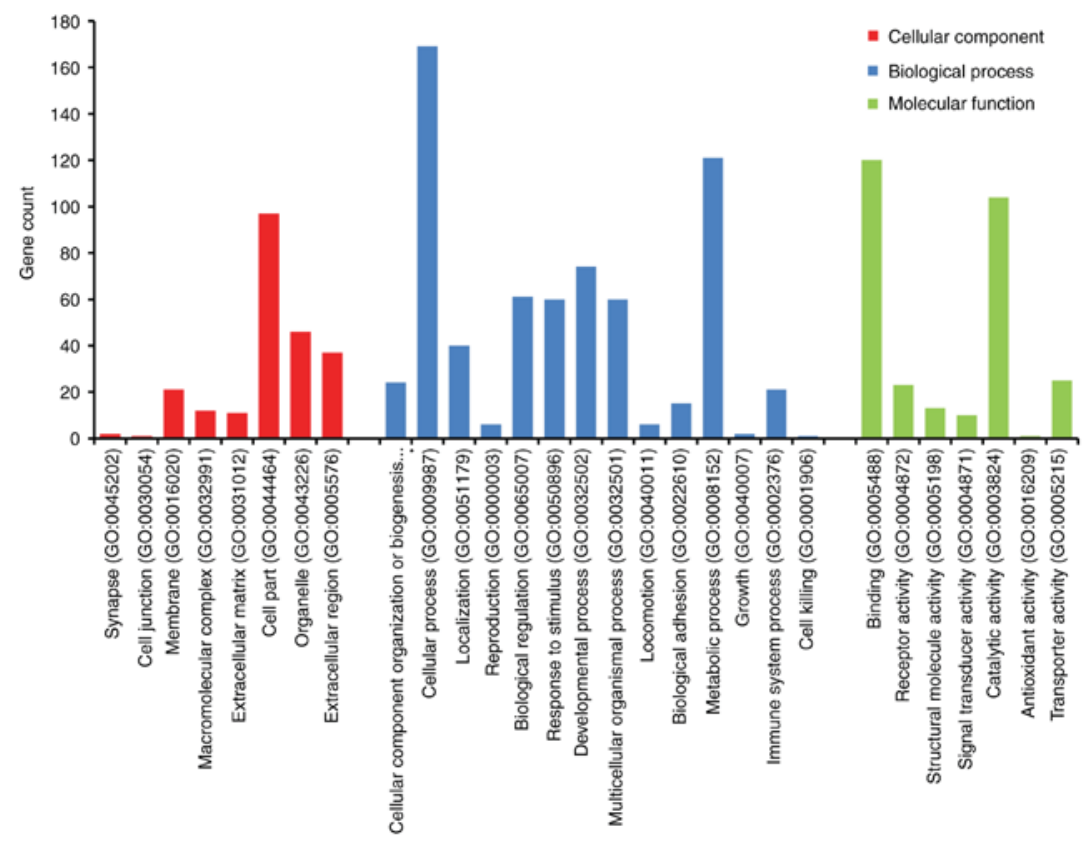

Figure 2. Diagram of GO analysis results classifying DEGs into three functional groups using Panther (http://www.pantherdb.org). GO, gene ontology; DEGs, differentially-expressed genes.

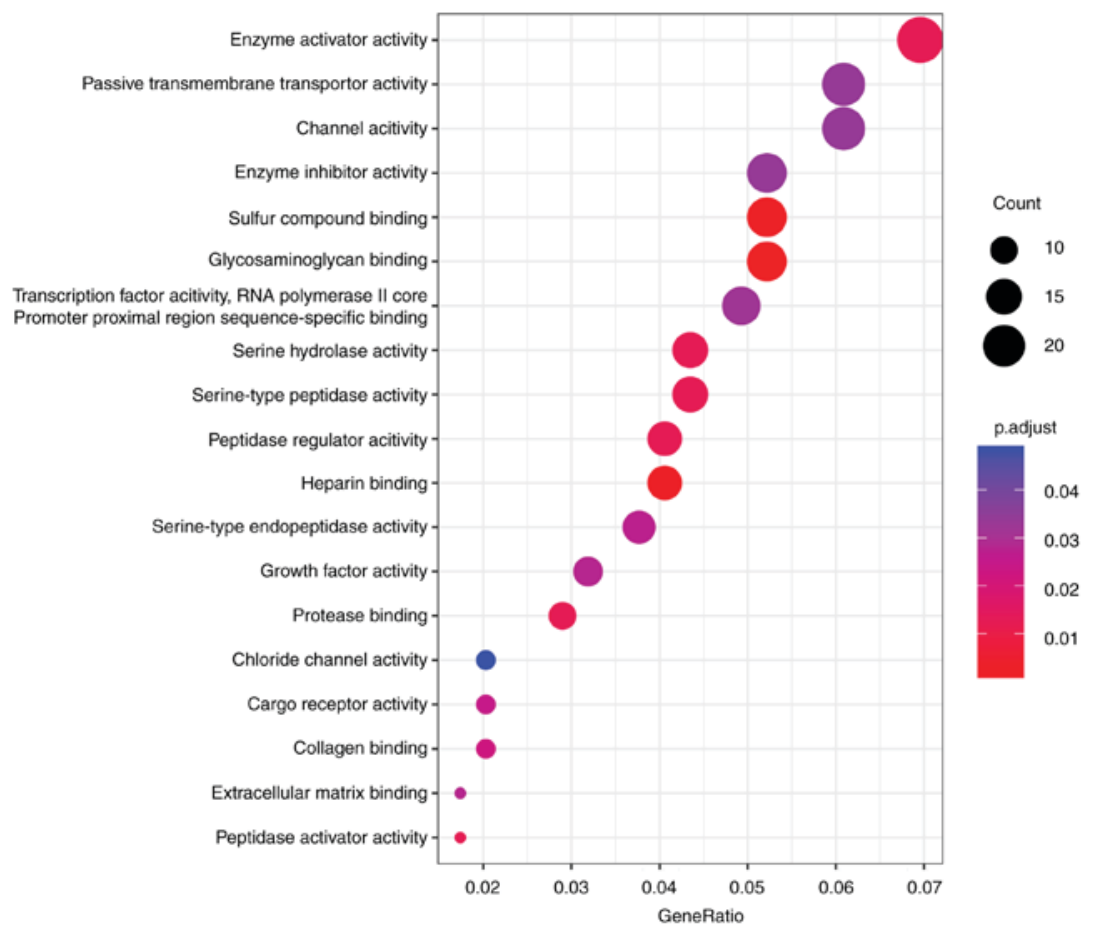

Figure 3. Representative enrichment GO terms for differentially expressed genes in papillary thyroid carcinoma. GO, gene ontology.

were downloaded from TCGA website and were analyzed using the same investigative approach (20). The results demonstrated that 186 upregulated and 184 downregulated genes were overlapping at two different sources (data not shown). Notably, 25 upregulated as well as 28 downregulated genes did not appear in the list, suggesting our results were credible.

RT-qPCR verification of selected DEGs. The expressions of 5 upregulated (COL10A1, COL1A1, COL8A1, LPAR5, NMU) and 3 downregulated (CFD, MMRN1, AGTR1) genes were validated using RT-qPCR assay of genetic material extracted from the peripheral blood samples of patients with PTC. The results demonstrated that gene expression of COL10A1, COL1A1, LPAR5, NMU, and CFD $(\mathrm{P}=0.0364, \mathrm{P}=0.0135$, $\mathrm{P}=0.0478, \mathrm{P}=0.0002, \mathrm{P}=0.0009$, PTC group vs. control group) were consistent with data from the bioinformatics analysis. There was no difference in the expression of COL8A1, MMRN1, and AGTR13 between the PTC and control groups (Fig. 6B). 
Table II. The significant enrichment analysis of DEGs in PTC.

\begin{tabular}{llrl}
\hline Term & \multicolumn{1}{c}{ Description } & Count & P-value \\
\hline GO:0005539 & Glycosaminoglycan binding & 18 & 0.0000004 \\
GO:1901681 & Sulfur compound binding & 18 & 0.0000018 \\
GO:0008201 & Heparin binding & 14 & 0.0000068 \\
GO:0008047 & Enzyme activator activity & 24 & 0.0000983 \\
GO:0016504 & Peptidase activator activity & 6 & 0.0001337 \\
GO:0002020 & Protease binding & 10 & 0.0001528 \\
GO:0008236 & Serine-type peptidase activity & 15 & 0.0001572 \\
GO:0017171 & Serine hydrolase activity & 15 & 0.0001805 \\
GO:0061134 & Peptidase regulator activity & 14 & 0.0002016 \\
GO:0005518 & Collagen binding & 7 & 0.0003936
\end{tabular}

DEGs, differentially expressed genes; PTC, papillary thyroid carcinoma; GO, gene ontology.

A

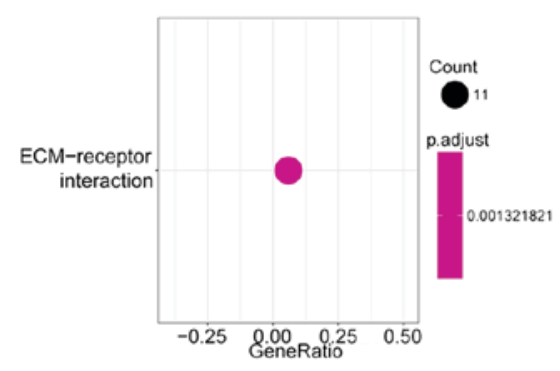

B

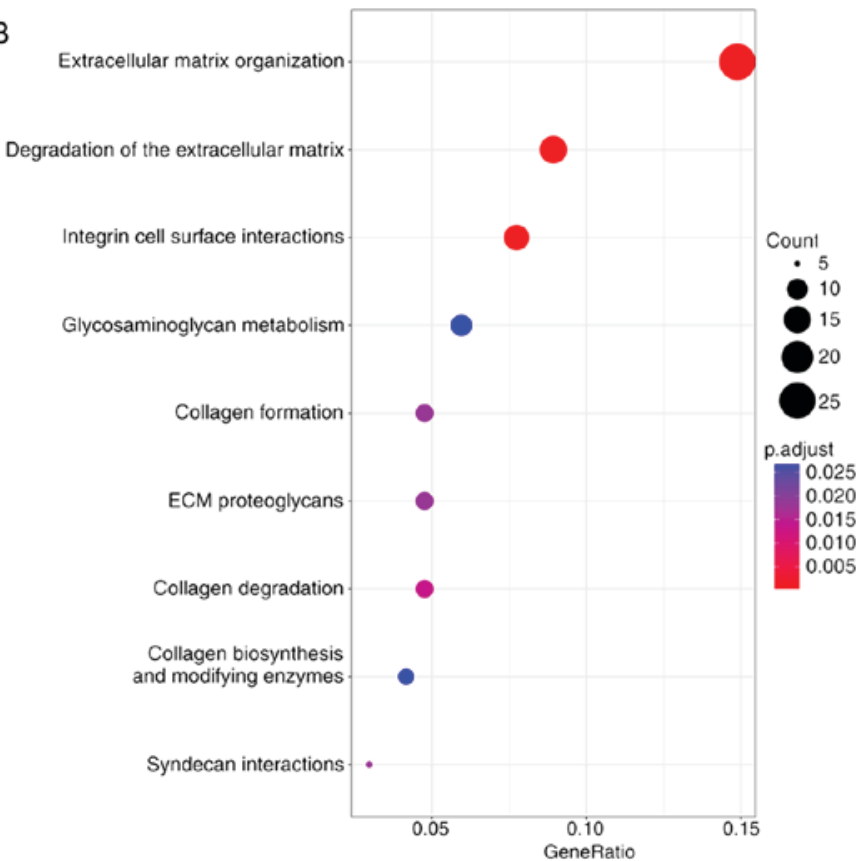

Figure 4. Representative enriched signaling pathways of differentially expressed genes in papillary thyroid carcinoma. (A) The DEGs had commonalities in hsa04512: ECM-receptor interaction pathway. (B) The reactome point of differentially expressed genes in PTC. DEGs, differentially expressed genes; PTC, papillary thyroid carcinoma.

\section{Discussion}

The present study identified 423 obvious DEGs between PTC tissues and normal controls, of which 211 were upregulated and 212 were downregulated. These 423 DEGs were then categorized into three groups (MF, BP and $\mathrm{CC}$ ) based on $\mathrm{GO}$ analysis. Results of GO and signaling pathway enquiry indicated that the DEGs were remarkably clustered in glycosaminoglycan binding, sulfur compound binding, heparin binding, enzyme activator activity, peptidase activator activity and hsa04512: ECM-receptor interaction. The reactome network of DEGs demonstrated that extracellular matrix organization and degradation as well as integrin cell surface interactions were key nodes of this network.

A PPI network was established using the selected DEGs and the most correlated 3 modules were selected for further analysis. Among these modules, 21 central node genes were present which were most associated with protease and $\mathrm{G}$-protein coupled receptor binding and peptidase regulator activity.

Consistent with the results obtained by the present study, other research groups also published the results of DEGs certification in PTC (11,18-21). For example, based on four datasets (GSE3467, GSE33630, GSE3678, GSE5315), Espinal-Enríquezet al (10) analyzed 64 healthy controls, 12 follicular thyroid carcinoma, 72 PTC and 11 anaplastic thyroid carcinoma samples, and reported there were an overall 503 upregulated and 457 downregulated genes in PTC. The topmost 10 dysregulated genes were GABRB2, HMGA2, PRR15, CHI3L1, ZCCHC12, TPO, DIO1, ADH1B, PKHD1L1, and TFF3. These DEGs were also identified in 
Table III. Signaling pathway enrichment analysis of DEGs in PTC.

\begin{tabular}{|c|c|c|c|c|}
\hline Pathway & Name & Gene Count & P-value & Genes \\
\hline 1474244 & Extracellular matrix organization & 25 & 0.000000002 & $\begin{array}{l}\text { BMP2/COL10A1/COL13A1/COL1A1/ } \\
\text { COL23A1/COL8A1/COL8A2/COL9A3/ } \\
\text { COMP/DCN/EFEMP1/FBLN1/FN1/ICAM1/ } \\
\text { ITGA2/KLK7/LAMB3/LRP4/MMP16/ } \\
\text { NCAM1/PRSS2/SDC4/SPP1/TIMP1/TNC }\end{array}$ \\
\hline 1474228 & $\begin{array}{l}\text { Degradation of the extracellular } \\
\text { matrix }\end{array}$ & 15 & 0.000000062 & $\begin{array}{l}\text { COL10A1/COL13A1/COL1A1/COL23A1/ } \\
\text { COL8A1/COL8A2/COL9A3/DCN/FN1/ } \\
\text { KLK7/LAMB3/MMP16/PRSS2/SPP1/ } \\
\text { TIMP1 }\end{array}$ \\
\hline 216083 & Integrin cell surface interactions & 13 & 0.000000103 & $\begin{array}{l}\text { COL10A1/COL13A1/COL1A1/COL23A1/ } \\
\text { COL8A1/COL8A2/COL9A3/COMP/FN1/ } \\
\text { ICAM1/ITGA2/SPP1/TNC }\end{array}$ \\
\hline 1442490 & Collagen degradation & 8 & 0.000134395 & $\begin{array}{l}\text { COL10A1/COL13A1/COL1A1/COL23A1/ } \\
\text { COL8A1/COL8A2/COL9A3/PRSS2 }\end{array}$ \\
\hline 3000178 & ECM proteoglycans & 8 & 0.000233271 & $\begin{array}{l}\text { COL1A1/COMP/DCN/FN1/ITGA2/LRP4/ } \\
\text { NCAM1/TNC }\end{array}$ \\
\hline 1474290 & Collagen formation & 8 & 0.000316917 & $\begin{array}{l}\text { COL10A1/COL13A1/COL1A1/COL23A1/ } \\
\text { COL8A1/COL8A2/COL9A3/LAMB3 }\end{array}$ \\
\hline 3000170 & Syndecan interactions & 5 & 0.000319611 & COL1A1/FN1/ITGA2/SDC4/TNC \\
\hline 1630316 & Glycosaminoglycan metabolism & 10 & 0.000587452 & $\begin{array}{l}\text { B4GALT6/CHST2/CSGALNACT1/DCN/ } \\
\text { GPC3/HS6ST2/LYVE1/OGN/PAPSS2/SDC4 }\end{array}$ \\
\hline 1650814 & $\begin{array}{l}\text { Collagen biosynthesis and } \\
\text { modifying enzymes }\end{array}$ & 7 & 0.000599989 & $\begin{array}{l}\text { COL10A1/COL13A1/COL1A1/COL23A1/ } \\
\text { COL8A1/COL8A2/COL9A3 }\end{array}$ \\
\hline
\end{tabular}

DEGs, differentially expressed genes; PTC, papillary thyroid carcinoma; ECM, extracellular matrix.

Table IV. Enrichment analysis of genes' function in modules 1-3.

\begin{tabular}{llrr}
\hline Term & \multicolumn{1}{c}{ Description } & Count & P-value \\
\hline GO:0002020 & Protease binding & 4 & 0.0000004 \\
GO:0001664 & G-protein coupled receptor binding & 5 & 0.0000018 \\
GO:0005201 & Extracellular matrix structural constituent & 3 & 0.0000068 \\
GO:0061134 & Peptidase regulator activity & 4 & 0.0000983 \\
GO:0008528 & G-protein coupled peptide receptor activity & 3 & 0.0001337 \\
GO:0001653 & Peptide receptor activity & 3 & 0.0001528 \\
GO:0071855 & Neuropeptide receptor binding & 2 & 0.0001572 \\
GO:0004866 & Endopeptidase inhibitor activity & 3 & 0.0001805 \\
GO:0061135 & Endopeptidase regulator activity & 3 & 0.0002016 \\
GO:0030414 & Peptidase inhibitor activity & 3 & 0.0003936
\end{tabular}

GO, gene ontology.

the present study and were primarily clustered in extracellular region and space and developmental process.

Yu et al (21) analyzed GSE3467 raw data including 9 PTC subjects and 9 paired normal tissues. They identified 1343 DEGs, of which, 651 were upregulated and 692 were downregulated, which were mainly enriched in complement and coagulation cascades as well as thyroid cancer pathways.
The most significant differentially expressed genes were MMP9, C3, PPARG, PAX8 and JUN. However, it is important to note that this study employed only one dataset. Similarly, Zhao et al (11) enrolled one dataset, GSE53157, containing 7 PTC specimens and 3 paired normal tissues, and ascertained 668 DEGs containing 262 upregulated genes and 406 downregulated genes, which were mainly enriched 


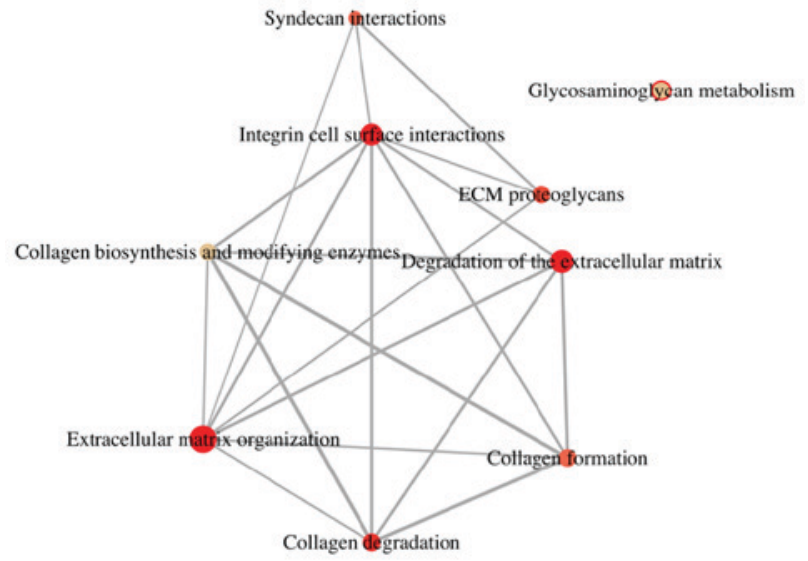

Figure 5. The reactome network of differentially expressed genes in papillary thyroid carcinoma.

in the signaling pathways related to programmed cell death, the p53 signaling cascades, the activities of protein kinase and transferase. They suggested that S100A6, MET and CDKN1C might have potential roles in the development of PTC.

Degradation of the extracellular matrix of adjacent tissues facilitates tumor invasion and metastasis (36). A recent study has identified that a regulatory loop exists between thyroid tumor cells, cancer associated fibroblasts (CAFs), collagen, and lysyl oxidase (Lox), which potentiates thyroid cancer progression (37). Qu et al (15) analyzed two microarray datasets (GSE3467 and GSE3678) and identified a total of 167 DEGs, which were associated with the regulation of plasma membrane and extracellular matrix.

The difference between our manuscript and the published papers on data mining for PTC is that the present study used R language and 'clusterProfile' in R for data processing, which can overcome some deficiencies such as data insufficiency caused by the update delay of some databases $(10,11,14,15,21)$. In addition, the datasets selected were all based on GPL570 platform (Affymetrix human genome U133 plus 2.0 array, Affymetrix; Thermo Fisher Scientific, Inc.) which is regarded as classic high-throughput expression microarray. This is helpful for the follow-up research, because by re-annotating and reassigning probe groups for functional regions of interest based on Affymetrix ${ }^{\circledR}$ GeneChip ${ }^{\circledR}$ technology, researchers can take advantage of the high volume of publicly available data to detect subtle changes in the region of interest likely to have phenotypical consequences in gene, transcript (isoform), untranslated region (UTR) and exon level with only minimal computational cost (38).

In the present study, reactome analysis indicated that the majority of the DEGs were primarily involved in extracellular matrix organization and degradation, integrin cell surface interactions, collagen degradation and collagen formation. The present study identified that COL10A1, COL13A1, COL1A1, COL8A1 and COL8A2 were upregulated in PTC, while COL9A3 and COL23A1 were downregulated. These DEGs work together to establish a network permissive of tumorigenesis (39), which requires further study, and hypothesize that this result will lead to the verification of additional therapeutic targets and biomarkers in PTC.
In the PPI network constructed in the present study, the second cluster consisted of CFD, ECM1, FN1, MMRN1, PROS1, SERPINA1 and TIMP1. Excluding CFD and MMRN1, all the genes were upregulated in PTC. ECM1 encodes a soluble protein that participates in angiogenesis and oncobiology (40). Kebebew et al (41) reported that ECM1 and TMPRSS4 were effective diagnostic markers of malignant thyroid nodules and differentiated thyroid cancers (DTC). FN1 regulates cell adhesion and migration processes (42). Its overexpression is an important determining factor in thyroid cancer aggression $(43,44)$. In a meta-analysis, SERPINA1 was identified as a single marker for PTC with 99\% accuracy (45). As a member of the TIMP gene family, TIMP1 encoded proteins that naturally inhibit MMP pathway resulting in the extracellular matrix degradation; a process closely associated with thyroid cancer invasiveness, migration and metastasis (10). In addition, TIMP1 encoded proteins promoted proliferation in various cell types and impeded cell apoptosis (46). TIMP1 mRNA had high inducibility to numerous cytokines and hormone stimulation (47).

In the third cluster of the PPI network, EDN3 encodes a protein belong to the endothelin family (48). Altered expression of this protein has been implicated in tumorigenesis (49). KISS1 is a metastasis suppressor gene (50). Its receptor, KISS1R, is a galanin-like $\mathrm{G}$ protein-coupled receptor that was demonstrated to be overexpressed in PTC and associated with MAP kinase activity (51). In DTC, KISS1 expression was conspicuously higher in aggressive and advanced tumors, which was moderately negatively correlated with tumor size (52). Lysophosphatidic acid receptor 5 (LPAR5) belongs to the rhodopsin class of $\mathrm{G}$ protein-coupled receptors (GPCR) superfamily that regulates various cellular processes engaged with tumor development (53). NMU encodes a member of the neuromedin family of neuropeptides that have effect in immune-mediated inflammatory diseases development; its overexpression was detected in tumors of renal, pancreatic and lung origins (54-57). The aforementioned analysis reminds us that these selected genes have not been the subjects of in-depth scientific investigation. Consequently, their relationship with PTC should be studied further.

The present study first verified the screened 423 DEGs with TCGA database and identified that 186 upregulated and 184 downregulated genes were overlapping in the two different sources (data not shown). The expressions of five upregulated (COL10A1, COL1A1, COL8A1, LPAR5, NMU) and three downregulated (CFD, MMRN1, AGTR1) genes were further validated using RT-qPCR of the peripheral blood samples of patients with PTC. These genes were selected as the majority of them were not previously reported in the literature. The results were consistent (5/8 genes were confirmed) with data from the bioinformatics analysis, suggesting our screened data were credible.

The present study was unable to validate all 21 key genes and could not solve the problem regarding histological, genetic, clinic biological characteristics and treatments of PTCs, primarily due to fund shortage. However among these genetic targets, LPAR5 was demonstrated to be involved in the pathogenesis of several types of cancer including melanoma, sarcoma, nasopharyngeal carcinoma $(53,58,59)$. LPAR5 is lysophosphatidic acid (LPA) receptor 5, encodes a member of the rhodopsin class of $\mathrm{G}$ protein-coupled transmembrane receptors (60). The 

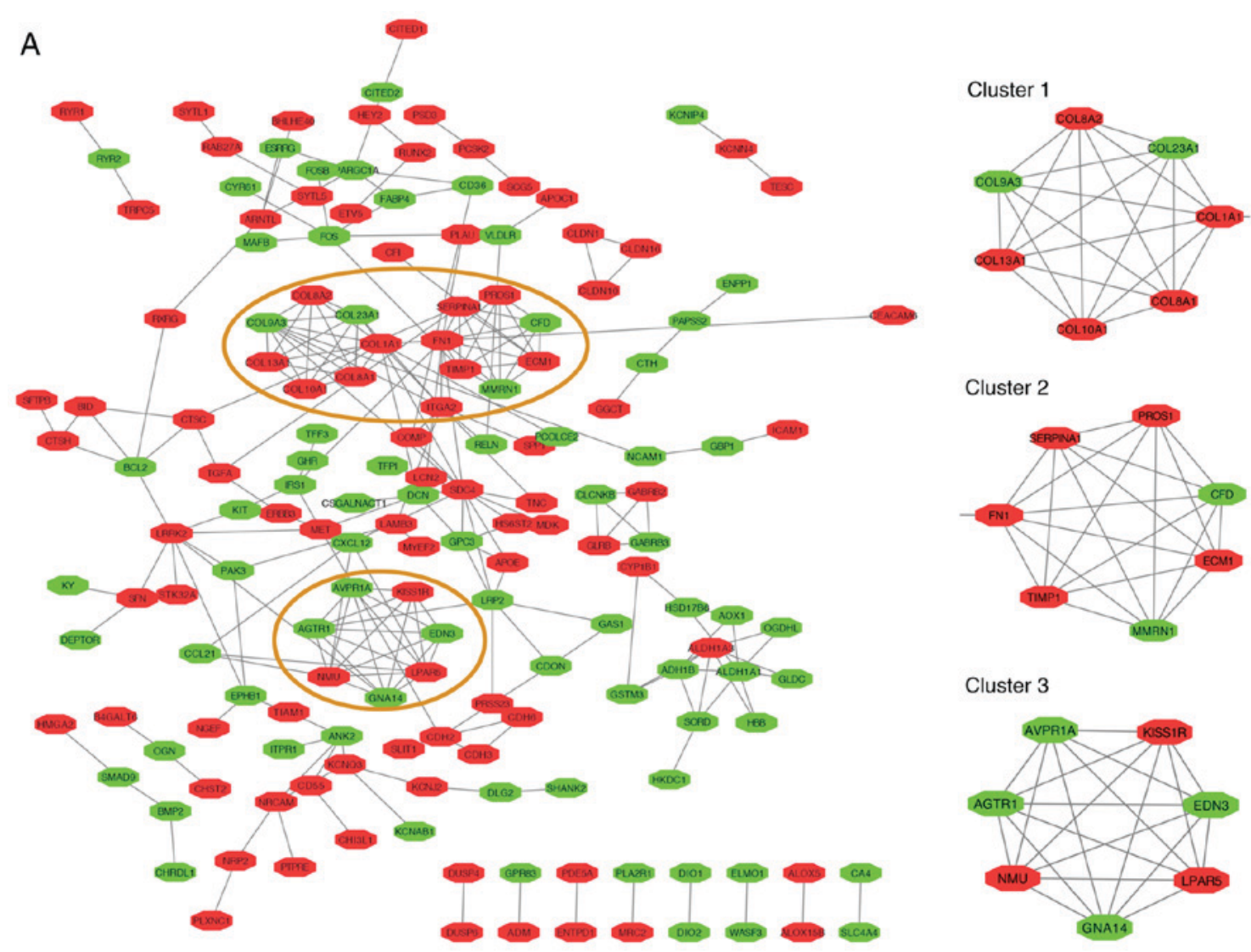

Cluster 3
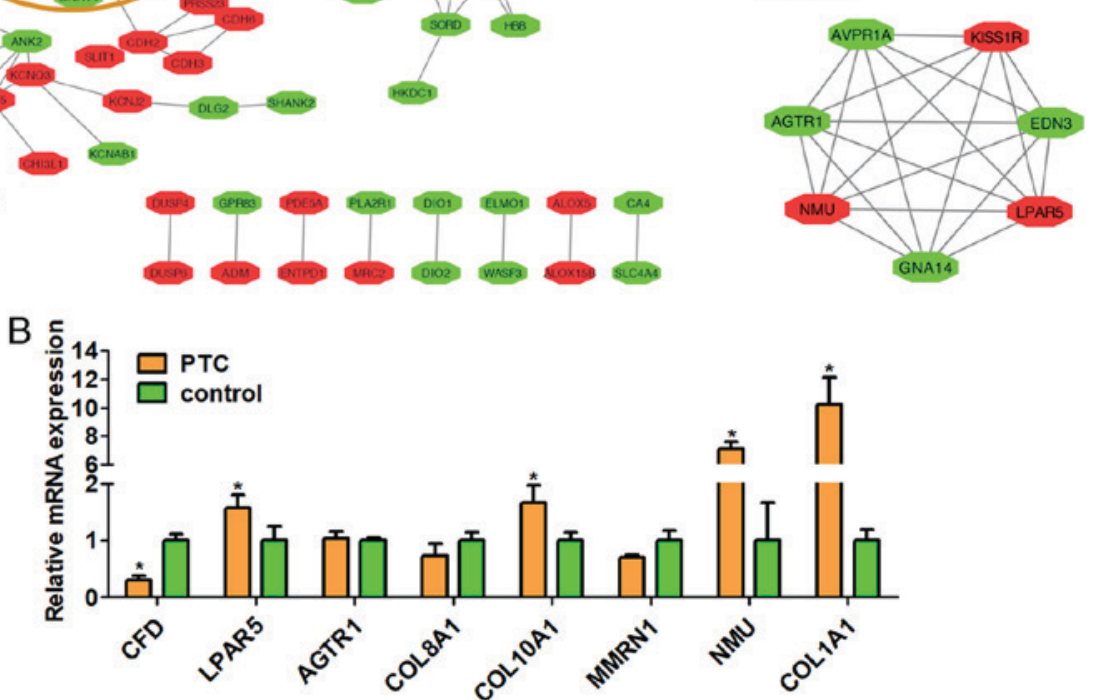

Figure 6. (A) Diagram of the protein-protein interaction network and three prominent modules (91 in red denote upregulated genes and 74 in green denote downregulated genes). The red circles represent the prominent clusters 1 to 3, and the right side are enlarged views of them. (B) Relative mRNA expressions of 8 differentially expressed genes isolated from peripheral blood samples of papillary thyroid carcinoma and control samples by reverse transcription-quantitative polymerase chain reaction analysis. CFD, complement factor D; LPAR5, Lysophosphatidic Acid Receptor 5; AGTR1, Angiotensin II Receptor Type 1; COL8A1, collagen type VIII $\alpha 1$ chain; COL10A1, collagen type X $\alpha 1$ chain; MMRN1, Multimerin 1; NMU, Neuromedin U; COL1A1, collagen type I $\alpha 1$ chain. ${ }^{*} \mathrm{P}<0.05$, PTC group vs. control group.

activated LPA stimulates cell proliferation, migration and survival (61). Differentially LPA production, receptor expression and signals contribute to cancer initiation, progression and metastasis (61). Database-based analysis (https://www. proteinatlas.org/) results also indicated that the prognosis of thyroid cancer patients with high expression level of LPAR5 was poor (https://www.proteinatlas.org/ENSG00000184574LPAR5/pathology/tissue/thyroid+cancer) $(62,63)$. Therefore, functional experiments of this gene including cell proliferation, migration, gain- and loss-of-function assays, should be conducted in the future.

In the present study, 423 DEGs were identified using three datasets from GEO with R programming language, and then filtered 392 gene nodes in DEGs PPI network, and 21 prominently altered key genes, which were significantly associated with extracellular matrix structural constituents and thyroid cancer invasiveness, migration and metastasis were selected. These candidate genes and pathways may have use as potential therapeutic targets in the future. These findings will expand the presently available knowledge regarding the etiology and essential molecular mechanisms at work in PTC progression. However, further experimentation on a larger clinical sample library should be used to verify these results.

\section{Acknowledgements}

Not applicable.

\section{Funding}

No funding was received. 


\section{Availability of data and materials}

The datasets used and/or analyzed during the current study are available from the corresponding author on reasonable request.

\section{Authors' contributions}

SL and HY designed the research, analyzed and interpreted the data, and wrote the manuscript. HY performed the RT-qPCR experiments. YY performed all the R language programming, data mining and visualizing tasks. All authors read and approved the final manuscript.

\section{Ethics approval and consent to participate}

The Ethics Committee of Guang'anmen Hospital approved the protocol of the present study and signed informed consent was obtained from all participants.

\section{Patient consent for publication}

All patients provided written informed consent for the publication of associated data.

\section{Competing interests}

The authors declare that they have no competing interests.

\section{References}

1. Nguyen QT, Lee EJ, Huang MG, Park YI, Khullar A and Plodkowski RA: Diagnosis and treatment of patients with thyroid cancer. Am Health Drug Benefits 8: 30-40, 2015.

2. Chen W, Zheng R, Baade PD, Zhang S, Zeng H, Bray F, Jemal A, Yu XQ and He J: Cancer statistics in China, 2015. CA Cancer J Clin 66: 115-132, 2016.

3. Haugen BR, Alexander EK, Bible KC, Doherty GM, Mandel SJ, Nikiforov YE, Pacini F, Randolph GW, Sawka AM, Schlumberger M, et al: 2015 American thyroid association management guidelines for adult patients with thyroid nodules and differentiated thyroid cancer: The american thyroid association guidelines task force on thyroid nodules and differentiated thyroid cancer. Thyroid 26: 1-133, 2016.

4. Siegel RL, Miller KD and Jemal A: Cancer Statistics, 2017. CA Cancer J Clin 67: 7-30, 2017.

5. Bisarro Dos Reis M, Barros-Filho MC, Marchi FA, Beltrami CM, Kuasne H, Pinto CAL, Ambatipudi S, Herceg Z, Kowalski LP and Rogatto SR: Prognostic classifier based on genome-wide DNA methylation profiling in well-differentiated thyroid tumors J Clin Endocrinol Metab 102: 4089-4099, 2017.

6. Fontaine JF, Mirebeau-Prunier D, Franc B, Triau S, Rodien P, Houlgatte R, Malthièry Y and Savagner F: Microarray analysis refines classification of non-medullary thyroid tumours of uncertain malignancy. Oncogene 27: 2228-2236, 2008.

7. Luzón-Toro B, Bleda M, Navarro E, Garcia-Alonso L, Ruiz-Ferrer M, Medina I, Martín-Sánchez M, Gonzalez CY, Fernández RM, Torroglosa A, et al: Identification of epistatic interactions through genome-wide association studies in sporadic medullary and juvenile papillary thyroid carcinomas. BMC Med Genomics 8: 83, 2015.

8. Handkiewicz-Junak D, Swierniak M, Rusinek D, Oczko-Wojciechowska M, Dom G, Maenhaut C, Unger K, Detours V, Bogdanova T, Thomas G, et al: Gene signature of the post-Chernobyl papillary thyroid cancer. Eur J Nucl Med Mol Imaging 43: 1267-1277, 2016.

9. Tomás G, Tarabichi M, Gacquer D, Hébrant A,Dom G, Dumont JE, Keutgen X, Fahey TJ III, Maenhaut C and Detours V: A general method to derive robust organ-specific gene expression-based differentiation indices: Application to thyroid cancer diagnostic. Oncogene 31: 4490-4498, 2012.
10. Espinal-Enríquez J, Muñoz-Montero S, Imaz-Rosshandler I, Huerta-Verde A, Mejía C and Hernández-Lemus E: Genome-wide expression analysis suggests a crucial role of dysregulation of matrix metalloproteinases pathway in undifferentiated thyroid carcinoma. BMC Genomics 16: 207, 2015.

11. Zhao M, Wang KJ, Tan Z, Zheng CM, Liang Z and Zhao JQ: Identification of potential therapeutic targets for papillary thyroid carcinoma by bioinformatics analysis. Oncol Lett 11: 51-58, 2016.

12. Guo Y, Bao Y, Ma M and Yang W: Identification of key candidate genes and pathways in colorectal cancer by integrated bioinformatical analysis. Int J Mol Sci 18: E722, 2017.

13. Zhang C, Peng L, Zhang Y, Liu Z, Li W, Chen S and Li G: The identification of key genes and pathways in hepatocellular carcinoma by bioinformatics analysis of high-throughput data. Med Oncol 34: 101, 2017.

14. Qiu WH, Chen GY, Cui L, Zhang TM, Wei F and Yang Y: Identification of differential pathways in papillary thyroid carcinoma utilizing pathway co-expression analysis. J BUON 21: 1501-1509, 2016.

15. Qu T, Li YP, Li XH and Chen Y: Identification of potential biomarkers and drugs for papillary thyroid cancer based on gene expression profile analysis. Mol Med Rep 14: 5041-5048, 2016.

16. Huang Y, Tao Y, Li X, Chang S, Jiang B, Li F and Wang ZM: Bioinformatics analysis of key genes and latent pathway interactions based on the anaplastic thyroid carcinoma gene expression profile. Oncol Lett 13: 167-176, 2017.

17. Qiu J, Zhang W, Xia Q, Liu F, Li L, Zhao S, Gao X, Zang C, Ge R and Sun Y: RNA sequencing identifies crucial genes in papillary thyroid carcinoma (PTC) progression. Exp Mol Pathol 100: 151-159, 2016.

18. Min XS, Huang P, Liu X, Dong C, Jiang XL, Yuan ZT, Mao LF and Chang S: Bioinformatics analyses of significant prognostic risk markers for thyroid papillary carcinoma. Tumour Biol 36: 7457-7463, 2015.

19. Chen W, Liu Q, Lv Y, Xu D, Chen W and Yu J: Special role of JUN in papillary thyroid carcinoma based on bioinformatics analysis. World J Surg Oncol 15: 119, 2017.

20. Cancer Genome Atlas Research Network: Integrated genomic characterization of papillary thyroid carcinoma. Cell 159: 676-690, 2014.

21. Yu J, Mai W, Cui Y and Kong L: Key genes and pathways predicted in papillary thyroid carcinoma based on bioinformatics analysis. J Endocrinol Invest 39: 1285-1293, 2016.

22. Vasko V,Espinosa AV, Scouten W, He H, Auer H, Liyanarachchi S, Larin A, Savchenko V, Francis GL, de la Chapelle A, et al: Gene expression and functional evidence of epithelial-to-mesenchymal transition in papillary thyroid carcinoma invasion. Proc Natl Acad Sci USA 104: 2803-2808, 2007.

23. Hilmarsdóttir B, Briem E, Sigurdsson V, Franzdóttir SR, Ringnér M, Arason AJ, Bergthorsson JT, Magnusson MK and Gudjonsson T: MicroRNA-200c-141 and $\Delta$ Np63 are required for breast epithelial differentiation and branching morphogenesis. Dev Biol 403: 150-161, 2015.

24. Barrett T, Wilhite SE, Ledoux P, Evangelista C, Kim IF, Tomashevsky M, Marshall KA, Phillippy KH, Sherman PM, Holko M, et al: NCBI GEO: Archive for functional genomics data sets-update. Nucleic Acids Res 41: D991-D995, 2013.

25. Ritchie ME, Phipson B, Wu D, Hu Y, Law CW, Shi W and Smyth GK: Limma powers differential expression analyses for RNA-sequencing and microarray studies. Nucleic Acids Res 43: e47, 2015.

26. Yu G, Wang LG, Han Y and He QY: ClusterProfiler: An R package for comparing biological themes among gene clusters. OMICS 16: 284-287, 2012.

27. Mi H, Huang X, Muruganujan A, Tang H, Mills C, Kang D and Thomas PD: PANTHER version 11: Expanded annotation data from Gene Ontology and Reactome pathways, and data analysis tool enhancements. Nucleic Acids Res 45: D183-D189, 2017.

28. Szklarczyk D, Morris JH, Cook H, Kuhn M, Wyder S, Simonovic M, Santos A, Doncheva NT, Roth A, Bork P, et al: The STRING database in 2017: Quality-controlled protein-protein association networks, made broadly accessible. Nucleic Acids Res 45: D362-D368, 2017.

29. Gautier L, Cope L, Bolstad BM and Irizarry RA: Affy-analysis of Affymetrix GeneChip data at the probe level. Bioinformatics 20: 307-315, 2004

30. Szklarczyk D, Franceschini A, Wyder S, Forslund K, Heller D, Huerta-Cepas J, Simonovic M, Roth A, Santos A, Tsafou KP, et al: STRING v10: Protein-protein interaction networks, integrated over the tree of life. Nucleic Acids Res 43: D447-D452, 2015. 
31. Shannon P, Markiel A, Ozier O, Baliga NS, Wang JT, Ramage D, Amin N, Schwikowski B and Ideker T: Cytoscape: A software environment for integrated models of biomolecular interaction networks. Genome Res 13: 2498-2504, 2003.

32. Deng Q, Li KY, Chen H, Dai JH, Zhai YY, Wang Q, Li N, Wang YP and Han ZG: RNA interference against cancer/testis genes identifies dual specificity phosphatase 21 as a potential therapeutic target in human hepatocellular carcinoma. Hepatology 59: 518-530, 2014.

33. Wang K, Niu Y, Wang Q, Liu H, Jin Y and Zhang S: Cloning and evaluation of reference genes for quantitative real-time PCR analysis in amorphophallus. Peer J 5: e3260, 2017.

34. Livak KJ and Schmittgen TD: Analysis of relative gene expression data using real-time quantitative PCR and the 2(-Delta Delta C(T)) method. Methods 25: 402-408, 2001.

35. Lefever S, Hellemans J, Pattyn F, Przybylski DR, Taylor C, Geurts R, Untergasser A, Vandesompele J and RDML RDML consortium: Structured language and reporting guidelines for real-time quantitative PCR data. Nucleic Acids Res 37: 2065-2069, 2009.

36. Lu P, Weaver VM and Werb Z: The extracellular matrix: A dynamic niche in cancer progression. J Cell Biol 196: 395-406, 2012.

37. Jolly LA, Novitskiy S, Owens P, Massoll N, Cheng N, Fang W, Moses HL and Franco AT: Fibroblast-mediated collagen remodeling within the tumor microenvironment facilitates progression of thyroid cancers driven by brafv600e and pten loss. Cancer Res 76: 1804-1813, 2016.

38. Saka E, Harrison BJ, West K, Petruska JC and Rouchka EC: Framework for reanalysis of publicly available affymetrix $(R)$ genechip(R) data sets based on functional regions of interest. BMC Genomics 18 (Suppl 10): S875, 2017.

39. Miyake M, Hori S, Morizawa Y, Tatsumi Y, Toritsuka M, Ohnishi S, Shimada K, Furuya H, Khadka VS, Deng Y, et al: Collagen type IV alpha 1 (COL4A1) and collagen type XIII alpha 1 (COL13A1) produced in cancer cells promote tumor budding at the invasion front in human urothelial carcinoma of the bladder. Oncotarget 8: 36099-36114, 2017.

40. Sercu S, Zhang L and Merregaert J: The extracellular matrix protein 1: Its molecular interaction and implication in tumor progression. Cancer Invest 26: 375-384, 2008.

41. Kebebew E, Peng M, Reiff E, Duh QY, Clark OH and McMillan A: ECM1 and TMPRSS4 are diagnostic markers of malignant thyroid neoplasms and improve the accuracy of fine needle aspiration biopsy. Ann Surg 242: 353-361; discussion 361-353, 2005.

42. Waalkes S, Atschekzei F, Kramer MW, Hennenlotter J, Vetter G, Becker JU, Stenzl A, Merseburger AS, Schrader AJ, Kuczyk MA and Serth J: Fibronectin 1 mRNA expression correlates with advanced disease in renal cancer. BMC Cancer 10: 503, 2010.

43. Sponziello M, Rosignolo F, Celano M, Maggisano V, Pecce V, De Rose RF, Lombardo GE, Durante C, Filetti S, Damante G, et al: Fibronectin-1 expression is increased in aggressive thyroid cancer and favors the migration and invasion of cancer cells. Mol Cell Endocrinol 431: 123-132, 2016.

44. Xia S, Wang C, Postma EL, Yang Y, Ni X and Zhan W: Fibronectin 1 promotes migration and invasion of papillary thyroid cancer and predicts papillary thyroid cancer lymph node metastasis. OncoTargets Ther 10: 1743-1755, 2017.

45. Vierlinger K, Mansfeld MH, Koperek O, Nohammer C, Kaserer K and Leisch F: Identification of SERPINA1 as single marker for papillary thyroid carcinoma through microarray meta analysis and quantification of its discriminatory power in independent validation. BMC Med Genomics 4: 30, 2011.

46. Porter JF, Shen S and Denhardt DT: Tissue inhibitor of metalloproteinase-1 stimulates proliferation of human cancer cells by inhibiting a metalloproteinase. Br J Cancer 90: 463-470, 2004.

47. Fagerberg L, Hallstrom BM, Oksvold P, Kampf C, Djureinovic D, Odeberg J, Habuka M, Tahmasebpoor S, Danielsson A, Edlund $\mathrm{K}$, et al: Analysis of the human tissue-specific expression by genome-wide integration of transcriptomics and antibody-based proteomics. Mol Cell Proteomics 13: 397-406, 2014.
48. Stanchina L, Baral V, Robert F, Pingault V, Lemort N, Pachnis V, Goossens $M$ and Bondurand N: Interactions between Sox10, Edn3 and Ednrb during enteric nervous system and melanocyte development. Dev Biol 295: 232-249, 2006.

49. Wiesmann F, Veeck J, Galm O, Hartmann A, Esteller M, Knuchel R and Dahl E: Frequent loss of endothelin-3 (EDN3) expression due to epigenetic inactivation in human breast cancer. Breast Cancer Res 11: R34, 2009.

50. Nash KT and Welch DR: The KISS1 metastasis suppressor: Mechanistic insights and clinical utility. Front Biosci 11: 647-659, 2006.

51. Ringel MD, Hardy E, Bernet VJ, Burch HB, Schuppert F, Burman KD and Saji M: Metastin receptor is overexpressed in papillary thyroid cancer and activates MAP kinase in thyroid cancer cells. J Clin Endocrinol Metab 87: 2399, 2002.

52. Savvidis C, Papaoiconomou E, Petraki C, Msaouel P and Koutsilieris M: The role of KISS1/KISS1R system in tumor growth and invasion of differentiated thyroid cancer. Anticancer Res 35: 819-826, 2015

53. Dong Y, Hirane M, Araki M, Fukushima N, Honoki K and Tsujiuchi T: Lysophosphatidic acid receptor-5 negatively regulates cell motile and invasive activities of human sarcoma cell lines. Mol Cell Biochem 393: 17-22, 2014.

54. Takahashi K, Furukawa C, Takano A, Ishikawa N, Kato T, Hayama S, Suzuki C, Yasui W, Inai K, Sone S, et al: The neuromedin U-growth hormone secretagogue receptor $1 \mathrm{~b} /$ neurotensin receptor 1 oncogenic signaling pathway as a therapeutic target for lung cancer. Cancer Res 66: 9408-9419, 2006.

55. Ketterer K, Kong B, Frank D, Giese NA, Bauer A, Hoheisel J, Korc M, Kleeff J, Michalski CW and Friess H: Neuromedin U is overexpressed in pancreatic cancer and increases invasiveness via the hepatocyte growth factor c-Met pathway. Cancer Lett 277: 72-81, 2009.

56. Harten SK, Esteban MA, Shukla D, Ashcroft M and Maxwell PH: Inactivation of the von hippel-lindau tumour suppressor gene induces neuromedin $U$ expression in renal cancer cells. Mol Cancer 10: 89, 2011.

57. Wang L, Chen C, Li F, Hua QQ, Chen S, Xiao B, Dai M, Li M, Zheng A, Yu D, et al: Overexpression of neuromedin $\mathrm{U}$ is correlated with regional metastasis of head and neck squamous cell carcinoma. Mol Med Rep 14: 1075-1082, 2016.

58. Lee SC, Fujiwara Y, Liu J, Yue J, Shimizu Y, Norman DD, Wang Y, Tsukahara R, Szabo E, Patil R, et al: Autotaxin and LPA 1 and LPA5 receptors exert disparate functions in tumor cells versus the host tissue microenvironment in melanoma invasion and metastasis. Mol Cancer Res 13: 174-185, 2015.

59. Yap LF, Velapasamy S, Lee HM, Thavaraj S, Rajadurai P, Wei W, Vrzalikova K, Ibrahim MH, Khoo AS, Tsao SW, et al: Down-regulation of LPA receptor 5 contributes to aberrant LPA signalling in EBV-associated nasopharyngeal carcinoma. J Pathol 235: 456-465, 2015.

60. Tsujiuchi T, Araki M, Hirane M, Dong Y and Fukushima N: Lysophosphatidic acid receptors in cancer pathobiology. Histol Histopathol 29: 313-321, 2014.

61. Mills GB and Moolenaar WH: The emerging role of lysophosphatidic acid in cancer. Nat Rev Cancer 3: 582-591, 2003.

62. Uhlen M, Zhang C, Lee S, Sjöstedt E, Fagerberg L, Bidkhori G, Benfeitas R, Arif M, Liu Z, Edfors F, et al: A pathology atlas of the human cancer transcriptome. Science. 357: eaan2507, 2017.

63. Uhlen M, Oksvold P, Fagerberg L, Lundberg E, Jonasson K, Forsberg M, Zwahlen M, Kampf C, Wester K, Hober S, et al: Towards a knowledge-based human protein atlas. Nat Biotechnol 28: 1248-1250, 2010.

This work is licensed under a Creative Commons Attribution-NonCommercial-NoDerivatives 4.0 International (CC BY-NC-ND 4.0) License. 